\title{
Characterisation of High Accuracy, Feedback Controlled, Adhesive Bonding
}

\author{
Rik Lafeber, Gerrit van den Bosch, Max Murre, Jitze Bassa, \\ Leo van Moergestel, and Erik Puik \\ HU University of Applied Sciences, Oudenoord 700, Utrecht, The Netherlands \\ erik.puik@hu.nl
}

\begin{abstract}
Adhesive bonding for industrial purposes has, over the last years, become more important compared to conventional joining methods, due to application in miniaturized systems where other ways of joining are too bulky. These days' industries have to deal with the challenge of applying adhesive bonds with small tolerances, while the geometrical dimensions of the applied adhesives change due to the curing process of the bond. This effect is called 'adhesive shrinkage'. This paper explains the effects of adhesive shrinkage, during bonding processes, on internal bond stress and the influences on position accuracy of the adherent parts. Effect of parameters like curing time, gap size between components and adhesive volume are studied. This is done by use of a test setup containing a manipulator with force sensor to simulate an industrial setup for the application of similar adhesive bonds. Results show that the gapsize has influence on shrinkage behaviour, whereas the adhesive volume, which defines the cross section area of the bond, is less important. Results can be used to develop a bonding procedure, which leads to an optimal balance between position accuracy and bond stress, thus guaranteeing a reliable and accurate bond in industrial manufacturing environments.
\end{abstract}

Keywords: adhesive bonding, force control, bond stress, position accuracy, process parameters, gap size, bond volume, curing time.

\section{Introduction}

Over the last decades, there is an increasing trend in using adhesive bonding for mechanical precision applications. An often-used bonding method for micro applications is to design form fit adhesive bonds [1]. Advantages of a form fitted adhesive bond are the ease of application and the high strength due to a large contact area [2]. Disadvantages are the high stresses in the bond and the unpredictable displacements during the bonding process [3].

It is generally known that most adhesives decrease in volume during the process of curing [1][4]. Holding the components very firmly during the curing process, to maintain the position accuracy, will typically result in highly stressed bonds, caused by adhesive shrinkage (Fig. 1) [5][6]. Releasing the components during the curing process will however effect unwanted displacement or position inaccuracy, mostly 
caused by shrinkage of the adhesive (Fig.2) [7][8]. In this paper, different bonding parameters, which have influence on this position accuracy and stress of the adhesive bond, have been studied.

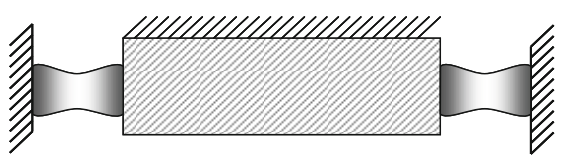

Fig. 1. Fixation of the components during the curing process of the adhesive causes high stress inside the adhesive bonds. Due to the high stress the bonds have a decreased life expectancy.

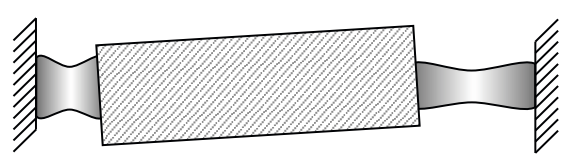

Fig. 2. If the part is released before the curing takes place, the components will suffer from loss of position accuracy. Depending on the geometry, there is no guarantee that the bonds will be more reliable than in the initial situation.

The aim of the project is to find an optimised procedure for the application of adhesive bonds, in which:

- The position of the components remains within accuracy specification or better, if possible;

- The stress in the joint is minimal;

- An optimal ratio between position accuracy and stresses in the bond is achieved.

The bonding procedure as tested in this project is based on the use of a feedbackcontrolled manipulator, combined with a force sensor. This enables optimal awareness of position, orientation and the applied forces during the curing process of adhesive bonding ( Fig.3). This objective can be applied up to six degrees of freedom, but was initially implemented with just one degree of freedom.

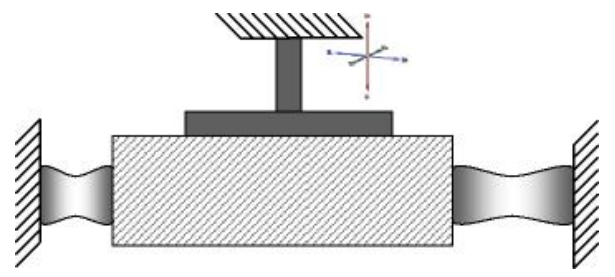

Fig. 3. A robotic manipulator actively controls the position of the part during the curing process. The position can be optimised so $1 /$ most important tolerances are kept but $2 /$ bond stresses are minimized.

A number of different parameters are found to have effect on shrinkage behaviour. These parameters therefore are suspected to worsen position tolerances of the components as well as stress-build-up in the bond. These parameters can be discriminated in process and environmental parameters. In this research, the following most important process parameters were varied, while the other parameters were kept constant:

- $\quad$ Size of the gap between the adherents;

- $\quad$ Curing time;

- $\quad$ Adhesive volume. 
Tests were performed on two extreme bonding situations. In these tests only a single bond was evaluated, being able to control the geometry and the tension of the bond, not suffering from dependencies of neighbouring bonds that might have conflicting behaviour. In the first 'Fixed-Position' test, the adherents were held firmly. This resulted in the highest stress value as marked by the triangular point in Fig. 4. In the second 'Zero-Force' test, the adherents are released, which shows the highest displacement value. This is marked by the round dot in Fig.4.

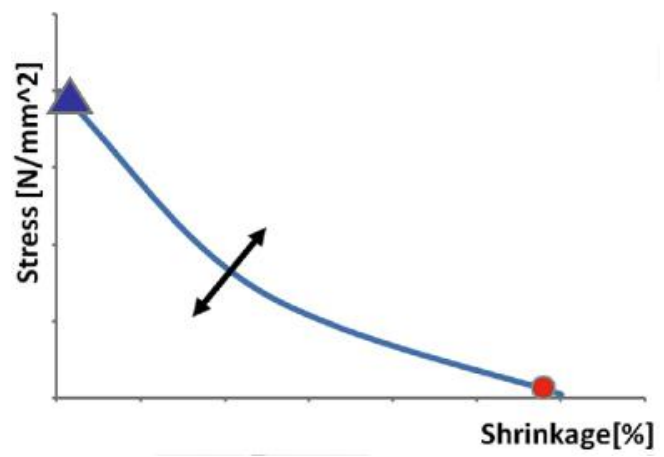

Fig. 4. Correlation between stress and shrinkage when applying adhesive bonds. By changing the way the parts are manipulated, any specific situation on the line can be selected. This enables influence on the ratio between position of the adherent and stress in the bond.

The stress in the bond is equal to the measured force divided by the effective cross section area of the adhesive bond. The shrinkage is measured by absolute distance, which is proportional to the span of the adhesive bond.

The adhesive used for the tests is Dymax Multicure 9-20801, which in these tests was cured by UV light with a wavelength of $365 \mathrm{~nm}$. Applicators or heat curing, which are used in the industry to increase the strength of the bond, are not applied. The reason for this is to prevent introduction of extra parameters in the test setup. This insures a higher reproducibility of the adhesive bond samples.

\section{Test Setup}

\subsection{Test Equipment}

The first experiment is to measure and control the force and displacement in both extreme points of the stress-shrinkage curve as shown in Fig. 4. The tests were performed on an industrial 3-axis gantry manipulator that was equipped with tools to hold the bonded parts in place and to continuously measure their positions. The axis' of the manipulator had stroke lengths of 540, 380 and $240 \mathrm{~mm}$ in respectively $\mathrm{x}, \mathrm{y} \& \mathrm{z}$ direction and were equipped with $1 \mu \mathrm{m}$ resolution encoders. This ensured a position accuracy of $<10 \mu \mathrm{m}$. The positioning action was applied at the most optimal position of the machine, in the centre and as low as possible on the machine bed, as shown in Fig. 5. This minimised orientation errors due to deformation of the robotic components. 


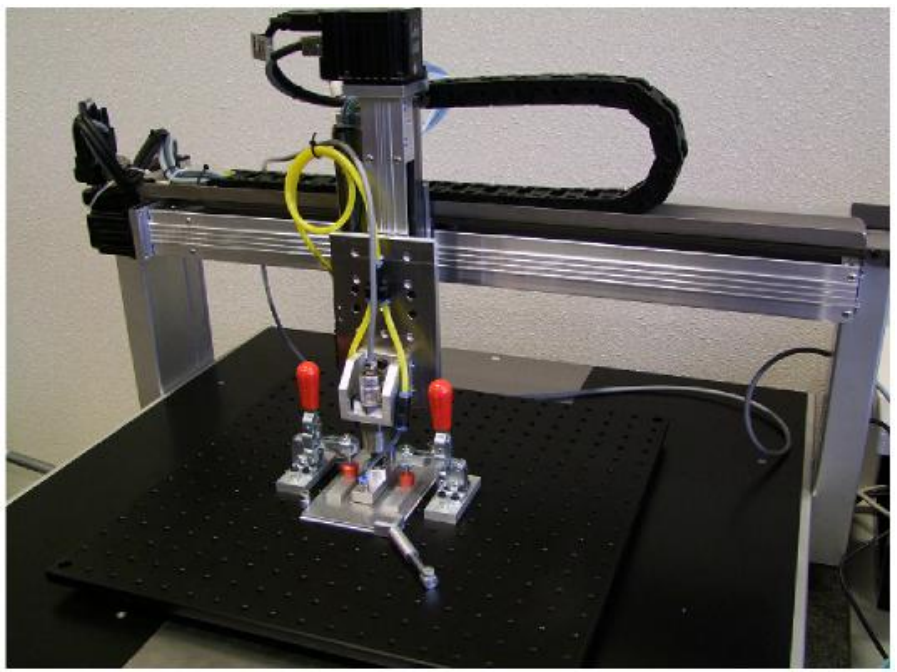

Fig. 5. The test setup as used for these tests consist of a gantry based robotic manipulator. One part is clamped mechanically, the other part is held using a vacuum gripper.

A 2-axis force sensor was placed between the bridge of the manipulator and a vacuum gripper as shown in Fig.6. The sensor is able to measure forces in $\mathrm{x}$ and $\mathrm{y}$ dimensions. The range of the force sensor was $0-100 \mathrm{~N}$ in both positive and negative direction with a non -linearity of $0,15 \%$.

The high-viscous adhesive was dispensed by a pressure-time dispensing system. The needle tip is pneumatically positioned above the gap, to ensure a repeatable dispensing process.

A camera system equipped with a photographic objective was mounted on a fixed stand and was able to monitor the bonding process accurately. The camera system was used to measure absolute displacement values. Combination of camera and lens provided a resolution of 2 pixels per micron, being able to perform measurements in the micron range.

\subsection{Calibration}

Before start of the tests, the system including camera and force sensor were calibrated. The force sensor was geometrically aligned perpendicular to the gantry system. The output signal of the force sensor was calibrated by use of reference masses.

The stiffness of the robotic manipulator, especially of the gantry construction and the actuators was measured. These parameters were to be used if needed to compensate the displacement due to the deformation of the robotic system when mechanically loaded. This occurs when the forces of the positioning process are transferred through the construction of the manipulator.

\subsection{Test Samples}

Standard test samples are used in all tests. The inner part, attached to the $\mathrm{x}$ - and $\mathrm{z}$ axis' was fixed to the vacuum gripper. The outer part is clamped to the base plate, 
which is mounted to the y-axis. The adhesive was dispensed in the middle of the gap between the inner and outer part. A Teflon sheet was applied to prevent sticking of the adhesive to the base plate (Fig. 6). The height of the inner part was chosen to hover above the Teflon sheet.

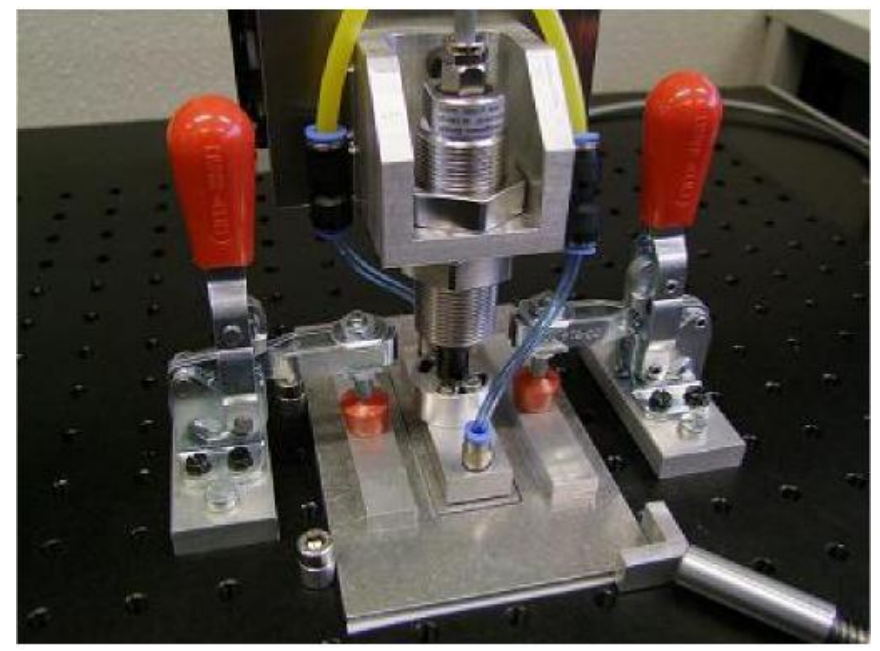

Fig. 6. Test sample clamped to the base plate

\subsection{Fixed-Position Test}

In the Fixed-Position test, the adherents, in this case the inner and outer part of the test sample, are kept in the same place during the entire curing process of the adhesive. To maintain the Fixed-Position, the manipulator was held at a certain encoder value. The correction, needed to compensate for the mechanical deformation of the gantry appeared relatively small. This eliminated the need for the feed forward correction of the gantry stability. The force was measured during the entire curing process in both $\mathrm{x}$ - and $\mathrm{y}$-direction.

\subsection{Zero-Force Test}

In the Zero-Force tests, the manipulator was actuated to suppress the accumulation of stress in the bond. The system was programmed to use feedback control based on input of the force sensor. In this way, the stress could be kept close to zero. The displacement of the adherents was measured by the movement of the encoder position and correlated to absolute distance by graphic distance measurement of the camera.

\section{$3 \quad$ Test Results}

The two extreme points of the stress-shrinkage curve, as explained in Fig.4, are found by performing the Zero-Force and Fixed-Position test repeatedly for the different 
bonding parameters, gap size, adhesive volume and curing time. A close-up of one of the adhesive joints from test results is shown in Fig. 7.

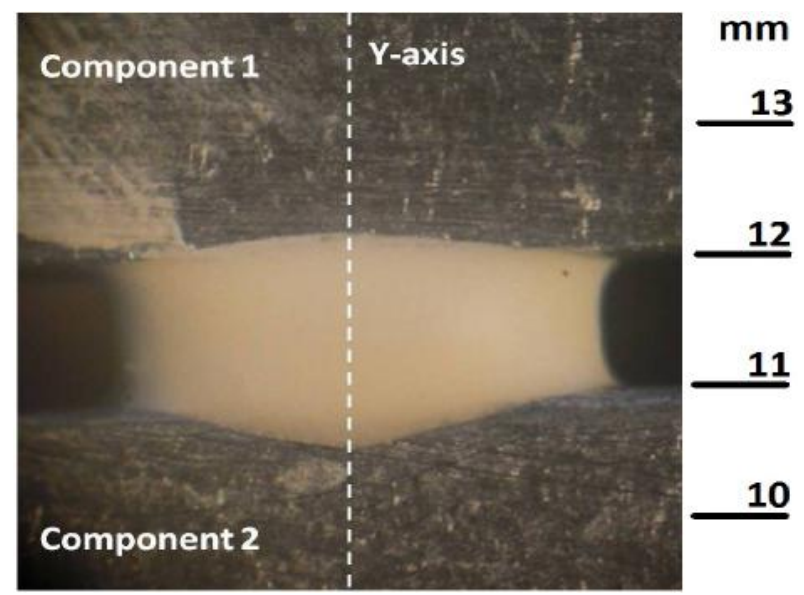

Fig. 7. Adhesive bond in one of the test samples. For this test the bond span is about one millimeter.

Fig. 8 shows the result of the Fixed -Position graphically. The accumulation of force in the bond is plotted against time. The maximum force arises at the end of the test. In this case the figure is plotted with 20 data points. At the end of the graph the bond is fully cured. This was verified by destructive tests on the first samples.

The graph shows that in this situation the accumulation of force takes place mainly within the first 30 seconds. Other adhesives will show a different time constant.

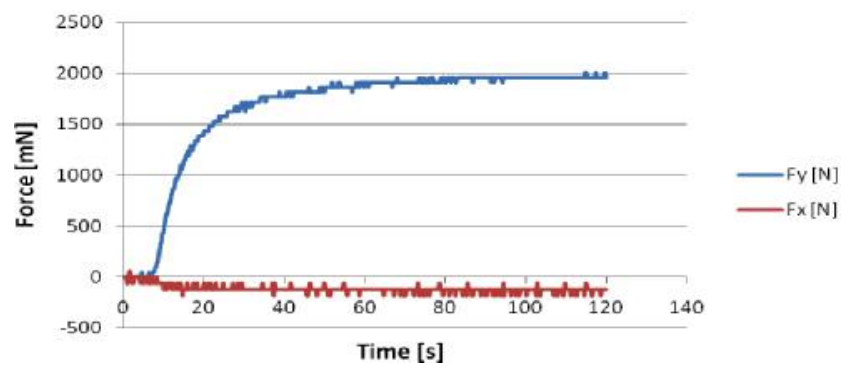

Fig. 8. Typical example of the results of the Fixed-Position test. Force accumulation develops in a natural way. In the right-angled direction the force remains low.

The result of a typical Zero-Force test is plotted in Fig. 9. Again, development of force is plotted against time. However, in this case the force is kept under a certain value by controlling the robotic manipulator with a feed back control loop. This enables the manipulator to move along with the moving parts. 

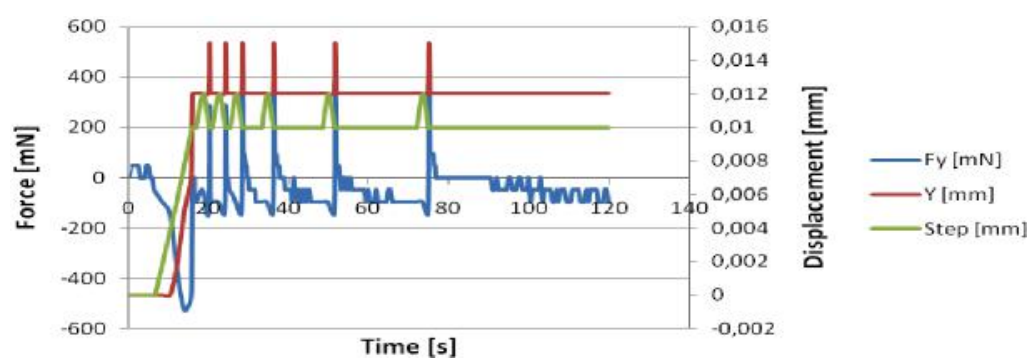

Fig. 9. Typical example result of the Zero-Force test. In this situation, all major adhesive shrinkage has taken place in the first 20 seconds. After this the control loop idles around a fixed value.

In the graph of Fig.9, $\mathrm{F}_{\mathrm{y}}$ represents the remaining force on the part and as such also on the adhesive bond. As can be seen, though considerable lower than the FixedPosition test, the force in the Zero-Force test is not completely eliminated. This is a result of the detection limit of the control hardware of the force sensor and discretization pollution of the digital control system.

Value $\mathrm{y}$ is the displacement measured by the motor encoder and since the forces in the system during these tests are low, can be considered as a realistic value for the actual displacement of the part. The maximum displacement was verified in to ways; using the control loop and by analysing the result of the camera. These measurements repeatedly showed results within small margins.

The 'step' curve is the cumulative value of all movement commands as registered by the Labview program. It represents the actual adhesive shrinkage of the bond. From the measurements at this point cannot be determined if the curve shows a linear, exponential or natural behaviour. Fact is that after an average time of 20 seconds, little movement was detected.

Both, Fixed-Position and Zero-Force tests are performed while varying the test parameters. The gap size was varied from $0,5 \mathrm{~mm}$ to $1,5 \mathrm{~mm}$ in three steps. The maximum force as determined by the Fixed-Position test and the maximum displacement determined by the Zero-Force test, are visualized in Fig. 10.

\section{Force vs shrinkage "gapsize"}

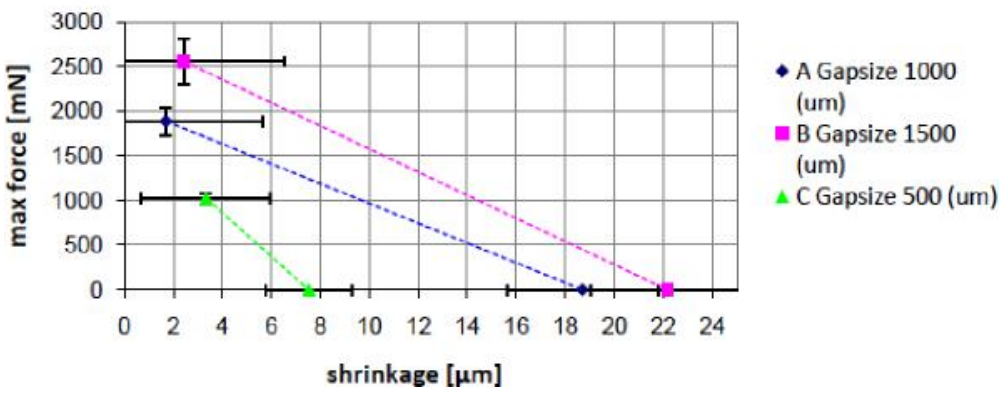

Fig. 10. Test result different gap sizes. In all cases the effect, force or shrinkage, increases when a larger span has to be bridged with the adhesive bond. Larger gaps lead to larger Forces and more adhesive shrinkage. 
The two points for each parameter-value are connected by a linear relation and are just for indication; they do not represent the real curve. The result of these 'Gap-Size' tests showed that both force and total adhesive shrinkage increase when the gap size increases.

Fig.11 shows the graph 'Force Versus Shrinkage' for three adhesive volumes, changing from $5 \mathrm{~mm}^{3}$ to $20 \mathrm{~mm}^{3}$. For this graph the same notice applies as to the previous; the lines only connect the end points but do not necessarily represent the points in between.

The effects of varying the adhesive volume on the maximum force and the shrinkage are minimal to none. Taken the deviation into account, adhesive volume has no significant influence on the maximum force and shrinkage.

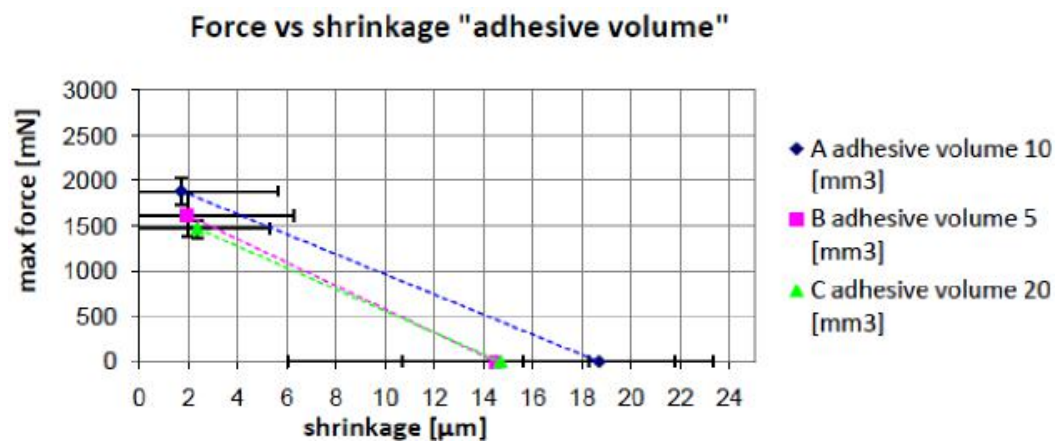

Fig. 11. Test results different adhesive volumes

Investigation of the effects of the curing time was done by performing the FixedPosition (maximum force) test in combination with a destructive test. Fig. 12 shows the development of force in time, of five measurements. Variation is limited between tests and the maximum force is reached around 250 seconds.

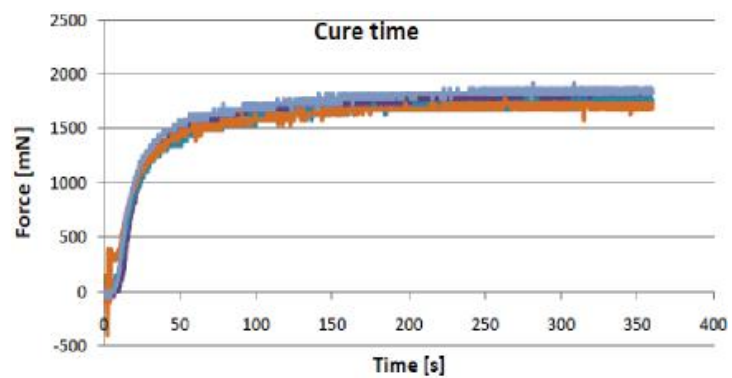

Fig. 12. Test results for curing time. A number of five measurements show good reproducibility. Around 250 seconds the adhesives may be considered fully cured.

\section{Conclusions}

The study of the parameter gap size shows a proportional relation between the gap sizes of the adherents with the force as well as the adhesive shrinkage. The conclusion 
can be drawn that the gap size has a high impact on the stress in the bond and the position accuracy of the components. A theoretical explanation for this behaviour could be a proportional shrinkage of the adhesive. A larger gap size will therefore result in a larger span of the adhesive material and thus more absolute shrinkage. However the fact that this leads to a higher force on the bond is not self-evident. The E-modulus of the adhesive is considerably lower than the metal of the parts. Based on this, some degree of suppression of the build-up of tension would be expected. Apparently, the elongation strength of the adhesive is dominant causing the force to increase.

The adhesive volume shows to be of less importance with respect to the force and adhesive shrinkage. Taking the variation into account, the results show no relation between adhesive volume and the measured force. This cannot be fully explained based on the results of these tests. Primarily, the fact that the adhesive shrinkage is comparable can be explained, a bulky adhesive dot could be considered as a number of small dots in parallel. All dots would have the same adhesive shrinkage. However, this would not explain that the total force doesn't increase. The force could expect to be proportional to the cross section area of the bond. A possible cause could be the fact that a larger does not tend to cure homogeneously, see also 'discussion'.

Curing times do not seen to have an effect on the force development and shrinkage during the curing process. With these tests, the intensity of the UV light source were not varied. Increased UV output might show effects on the process.

Destructive tests also show a positive effect on the end strength of the bond at longer curing times. For high filled industrial adhesives, like Dymax Multicure 920801 , it takes minutes to hours to cure the bond fully. To get the best results it is advised to use a post-cure process.

\section{Discussion}

Destructive tests with short curing times have demonstrated that the surface of the adhesive joint cures first and forms a hard and rigid shell. The adhesive material underneath the shell appears to cure more slowly. This can be explained by the decreased intensity of the UV light caused by the 'barrier' formed by the shell itself. 'Deeper' in the adhesive dot, the curing process takes a slower pace leading to an inhomogeneous curing process. When the shell is solidified, the core is still fluid and adapts is shape to the (already deformed shell). Some moments later the core starts to solidify, causing even more shrinkage on the inside of the bond. This process is very dependent on the physics of the illumination. In this project, this was not investigated.

Secondly, performing the Zero-Force tests led to the finding that implied movements of the adherents during the curing process, in some circumstances had negative influence on the bond strength. The effects appeared after complete curing of the adhesive bonds. It is important to understand the effects of movement during the curing process; it could subvert the results of the Zero-Force test.

Further studies must be performed in order to find an optimal ratio between position accuracy and stresses in the bond. Therefore, it is necessary to investigate the entire relation of stress-build-up and adhesive shrinkage as shown in Fig. 4. The data points in between can be found by defining a new test that combines elements from 
the Fixed -Position and the Zero-Force test. The most optimal ratio between accuracy and stress can be found once the whole stress-shrinkage curve is visualized. Further, this information will lead to a bonding strategy for industrial applications to offer maximum-performance-bonds within the boundaries of accuracy and stress.

For further investigations it is recommended to implement a position sensor for direct feedback to the controller instead of getting position information via motor encoder and camera. This sensor will provide position information with a higher resolution and accuracy so the robotic movements will allow smoother position control, reducing side effects of the manipulative actions.

Acknowledgments. The authors would like to thank the Knowledge Centre at the HU University Utrecht, the companies Adimec, Tegema and MA3Solutions for their support in knowledge and equipment. Much gratitude is afforded for the MicroNed program for funding this research (www.microned.nl).

\section{References}

1. Pocius, A.: Adhesion and adhesives technology: an introduction, p. 319. Hanser Gardner Pubns. (2002)

2. Diaz, A.D., Hadj-Ahmed, R., Foret, G.: Stress analysis in a classical double lap, adhesively bonded joint with a layerwise model. International Journal of É (2009)

3. Vrenken, J.: De invloed van de E-modulus op de sterkte van gelijmde overlapverbindingen

4. Pocius, A.: The mechanics of adhesion, p. 790. Elsevier Science (2002)

5. Neves, A., Coutinho, E., Poitevin, A., Van der Sloten, J.: Influence of Joint component mechanical properties and adhesive layer thickness on stress distribution in micro-tensile bond strength specimens. Dental Materials (2009)

6. Vrenken, J.: De invloed van de E-modulus op de sterkte van gelijmde overlapverbindingenHet berekenen van de sterkte van een lijmverbinding is nog altijd niet goed É. Constructeur (2006)

7. van Straalen, I.: Development of Design Rules for Structural Adhesive Bonded Joints-A Systematic Approach, p. 167 (2001)

8. Koster, M.P.: Constructieprincipes, p. 554 (2008) 